This is an author produced version of a paper published in Journal of Fish Biology.

This paper has been peer-reviewed but may not include the final publisher proof-corrections or pagination.

Citation for the published paper:

T. Backström, E. Brännäs, J. Nilsson and C Magnhagen. (2014) Behaviour, physiology and carotenoid pigmentation in Arctic charr Salvelinus alpinus.

Journal of Fish Biology. Volume: 84, pp 1-9.

http://dx.doi.org/:10.1111/jfb.12240.

Access to the published version may require journal subscription.

Published with permission from: Wiley.

Standard set statement from the publisher:

This is the accepted version of the following article: Backström, T., Brännäs, E., Nilsson, J. and Magnhagen, C. (2014), Behaviour, physiology and carotenoid pigmentation in Arctic charr Salvelinus alpinus. Journal of Fish Biology, 84: 1-9, which has been published in final form at http://dx.doi.org/:10.1111/jfb.12240.

Epsilon Open Archive http://epsilon.slu.se 


\title{
Behaviour, physiology and carotenoid pigmentation in Arctic charr Salvelinus alpinus
}

\author{
T. Backström ${ }^{1}$, E. Brännäs, J. Nilsson, and C. Magnhagen \\ Dept. of Wildlife, Fish, and Environmental Studies, Swedish University of Agricultural \\ Sciences, Umeå, Sweden
}

The behaviour during an exploration task and the stress response to a confinement stress of Arctic charr Salvelinus alpinus were evaluated. Behaviour of individuals during 90 minutes of exploration was classified into high and low activity. High-activity individuals had higher plasma cortisol levels following stress compared to low-activity individuals. This indicates that high- and low- activity individuals correspondg to reactive and proactive stress coping style. Further, a pigmentation analysis showed that high-activity individuals had a higher number of carotenoid spots $\mathrm{cm}^{-2}$ than low-activity individuals. Thus, carotenoid pigmentation, as melanin pigmentation in other salmonids, could be linked to stress coping style in S. alpinus.

Keywords: activity: behaviour; confinement stress; stress coping style

Journal of Fish Biology (2014) 84, 1-9

doi:10.1111/jfb.12240, available online at

http://onlinelibrary.wiley.com/doi/10.1111/jfb.12240/epdf

\section{INTRODUCTION}

Individual differences in animal behaviour have been shown during the last decades to be consistent across context and time and thus individuals express personality (Réale et al., 2007). This has been described under several different synonyms including behavioural syndromes (Sih et al., 2004), personality (Gosling, 2001), and temperament (Boissy, 1995). Another similar categorization of animals has been based on the different individual responses to stress. Individual stress responsiveness is shown in both behavioural and physiological differences which can be defined as stress-coping styles, and are generally divided into proactive and reactive coping style (Koolhaas et al., 1999, 2007). Proactive animals behave more aggressively, are more active, and readily form routines compared to reactive animals. In response to a stressor, proactive animals have a lower hypothalamicpituitary-adrenal (HPA) axis reactivity leading to a lower increase of glucocorticoids and a higher sympathetic activity leading to a higher increase of noradrenaline and adrenaline in blood compared to reactive animals.

In recent years, studies on teleost fish have established the existence of stress-coping styles in several different species such as halibut Hippoglossus hippoglossus (L. 1758) (Kristiansen \& Fernö, 2007), Nile tilapia Oreochromis niloticus (L. 1758) (Barreto \& Volpato, 2011) and Senegalese sole Solea senegalensis Kaup 1858 (Silva et al., 2010). Additionally, salmonid species have also been examined thoroughly. For instance, in brown

\footnotetext{
${ }^{1}$ Author to whom correspondence should be addressed. Tel.: +46 9078685 14; email:

Tobias.Backstrom@slu.se
} 
trout Salmo trutta L. 1758 individuals clustered into two separate stress-coping styles based on the physiological plasma levels of noradrenaline and adrenaline post confinement and behaviour during hypoxia (Brelin et al., 2005). Similarly, in rainbow trout Oncorhynchus mykiss Walbaum 1792) aggression, dominance and the stress hormone cortisol differed between individuals and resulted in two distinct coping styles (Schjolden et al., 2005a). Further, two selected strains of rainbow trout representing high (HR) and low responders (LR) with respect to cortisol release following a standardized confinement stress were developed and studied by Pottinger and Carrick (1999). Over a series of experiments the strains were shown to correspond to reactive and proactive stress coping strategy (Øverli et al., 2007).

In a recent study of the HR and LR strains of $O$. mykiss, melanin-based pigmentation was negatively correlated with stress responsiveness, with the LR trout having more melanin spots thanthe HR trout (Kittilsen et al., 2009). The same negative correlation between melanin-based spots and stress responsiveness was also found in Atlantic salmon Salmo salar L. 1758 (Kittilsen et al., 2009). Thus, it appears that melanin pigmentation and stress responsiveness are coupled in salmonid species in general. The same pattern is apparent in vertebrates (Ducrest et al., 2008), with darker animals being less sensitive to stress. The physiological link between melanin pigmentation and stress responsiveness is that both are affected by proopiomelanincortin (POMC) (Ducrest et al., 2008). In Arctic charr Salvelinus alpinus (L. 1758), however, the hypothesis that darker animals are more stress resilient does not appear to be valid. In interacting S. alpinus, the subordinates are darker and have higher plasma levels of the stress hormone cortisol (Höglund et al., 2000, 2002). Furthermore, $S$. alpinus differ from other salmonid species in life history, social behaviour and pigmentation (Klemetsen et al., 2003). One of the most apparent differences is the carotenoid-based pigmentation in S. alpinus. In several studies of teleosts, carotenoid-based pigmentation and physical prowess have been positively linked (Nicoletto, 1991; Nicoletto \& Kodric-Brown, 1999; Guderley \& Couture, 2005; Kodric-Brown \& Nicoletto, 2005). Based on this, an investigation was performed to see if stress-coping styles in S. alpinus are correlated to carotenoid-based pigmentation. Two hypotheses were formed: (1) behaviour and stress responsiveness are correlated in $S$. alpinus as in other salmonids and thus resulting in stress coping styles and (2) the resulting stress coping styles from hypothesis 1 are correlated (negatively or positively) with carotenoid-based pigmentation. Behaviour was monitored during an exploration task, plasma cortisol was measured after confinement stress and images analysed for carotenoid-based pigmentation.

\section{MATERIAL AND METHODS}

\section{EXPERIMENTAL ANIMALS AND LOCATION}

This study was carried out on 26 months old juvenile Arctic charr from 125 full sibling groups of the seventh generation of the Swedish S. alpinus breeding programme [Arctic superior, for details on the programme see Nilsson et al. (2010)], with a body mass $\left(\mathrm{M}_{\mathrm{B}}\right)$ of $965.6 \pm 352.8 \mathrm{~g}$ (mean \pm S.D., $\mathrm{n}=60)$ and a fork length $\left(\mathrm{L}_{\mathrm{F}}\right)$ of $371.6 \pm 44.9 \mathrm{~mm}$ (mean \pm S.D., n=60). Prior to the experiment, the S. alpinus were kept at the stocking facilities at the Fisheries Board Research Station in Kälarne, Sweden, and fed commercial pellets (Biomar, $4 \mathrm{~mm}$, www.biomar.com) at 1.0-1.5\% of body mass per day depending on temperature. The photoperiod was set to 10L:14D dark and temperature ranged from 2 to ${ }^{\circ} \mathrm{C}$. The experiment was performed in May 2011. 


\section{EXPERIMENTAL PROTOCOL}

At the start of the experiment, S. alpinus from the holding stock were anesthetized with tricaine methanesulfonate (MS-222, $0.15 \mathrm{~g}^{-1}$ ), weighed and measured, and the left side of each $S$. alpinus was photographed using a Canon EOS 500D digital camera

(www.canon.com) under ambient light kept constant throughout the session. S. alpinus were classified into one of three different size categories, small $(591.9 \pm 149.9 \mathrm{~g}, 322.0 \pm 28.1 \mathrm{~mm}$, $\mathrm{n}=19)$, medium $(922.1 \pm 76.1 \mathrm{~g}, 376.3 \pm 12.3 \mathrm{~mm}, \mathrm{n}=18)$, or large $(1308.3 \pm 256.8 \mathrm{~g}, 408.9 \pm$ $32.1 \mathrm{~mm}, \mathrm{n}=23$ ), for the following experiments. Individual $S$. alpinus were moved to isolation overnight in an aquarium prepared for an open field test. The aquaria were of two different sizes $(73 \times 40 \times 49 \mathrm{~cm}, 143 \mathrm{~L}$; and $71 \times 49.5 \times 49 \mathrm{~cm}, 172 \mathrm{~L})$ and supplied with water at ambient temperature from a nearby lake (also used for the holding stock) and the same photoperiod as in holding tanks (10L:14D). Each aquarium was separated into two compartments of equal size; one compartment was covered in black plastic, thus being dark, whereas the other compartment was exposed to light. The two compartments were connected by a opaque screen with a round opening ( $10 \mathrm{~cm}$ diameter) in the middle, covered by a sliding trapdoor. Initially, the S. alpinus were placed in the dark compartment. The second day, the trapdoor was removed and latency to enter the lit area and number of movements between the dark and the lit compartment (activity) was observed for $90 \mathrm{~min}$. The observers were sitting motionless $c .2 \mathrm{~m}$ from the aquaria, and took care not to disturb the fish during the behavioural test. During the third day, S. alpinus were exposed to an acute confinement stress test. S. alpinus were placed in one of three different confinement aquaria based on size of individual S. alpinus $(27.5 \times 18 \times 17 \mathrm{~cm}$, filled with 31 water for small size, $\mathrm{n}=19 ; 38 \times 26$ x $23.5 \mathrm{~cm}$, filled with 41 water for medium size, $\mathrm{n}=18 ; 42 \times 31$ x $25 \mathrm{~cm}$, filled with 51 water for large size, $\mathrm{n}=23$ ). After $30 \mathrm{~min}, \mathrm{~S}$. alpinus were sacrificed with a blow on the head, and blood was sampled. The blood was subsequently centrifuged, the plasma collected and stored at $-70{ }^{\circ} \mathrm{C}$ until further analysis.

\section{IMAGE ANALYSIS}

Photographs were analysed for carotenoid-based pigmentation, i.e. light colouration, with the free software ImageJ (Rasband, W.S., ImageJ, U. S. National Institutes of Health, Bethesda, Maryland, USA, http://imagej.nih.gov/ij/, 1997-2012.). Each side, from gills to tail, was divided into eight separate segments based on the visual cues of fins, gills, and lateral line (Fig. 1). The number of spots per segment was counted using the plugin PointPicker (http://bigwww.epfl.ch/thevenaz/pointpicker/) by an observer not involved in the behavioural monitoring. The area for each segment was determined via rectangular and triangular areas using the existing scale tool in ImageJ, based on the known $\mathrm{L}_{\mathrm{F}}$ of each individual. The end product used for analysis was number of spots $\mathrm{cm}^{-2}$. This procedure was done twice with strongly correlated results.

\section{RADIOIMMUNOASSAY}

Plasma cortisol levels were analysed using a radioimmunoassay (Pottinger \& Carrick, 2001). Each sample was run in a single assay with a minimal detection limit of $0.1 \mathrm{ng} \mathrm{ml}^{-1}$ and an 
intra-assay coefficient of variation $(\mathrm{C}: \mathrm{V})$ of $5.2 \%$. The cortisol antibody (ABIN108346) was a rabbit polyclonal (www.antibodies-online.com).
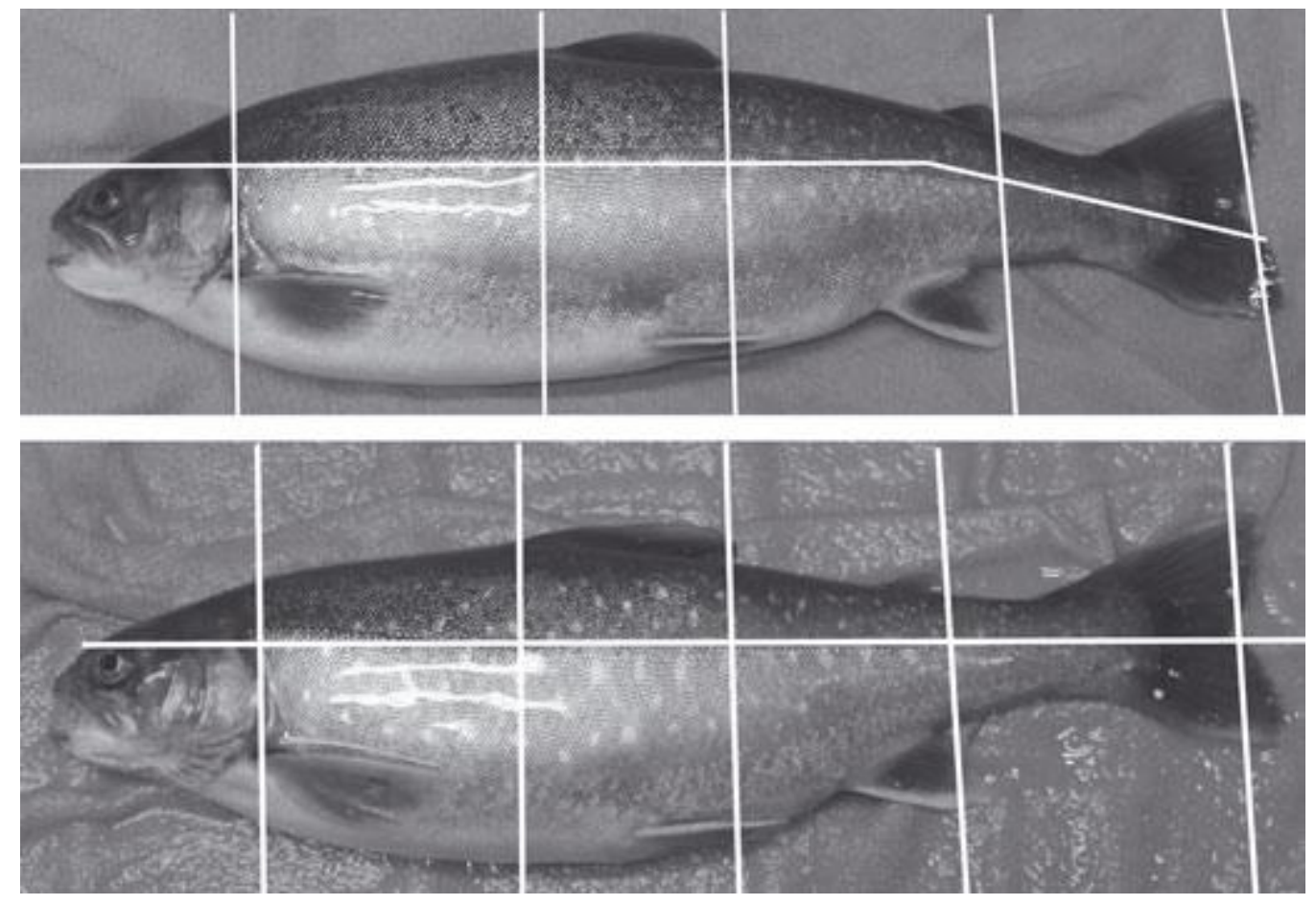

Fig. 1. Schematic representations of the areas that were used for the pigmentation counting. The top Salvelinus is representative of low pigmentation and the bottom S. alpinus is representative of high pigmentation.

\section{STATISTICAL ANALYSIS}

Two-tailed Spearman correlations were used to compare the aggregate as well as the individual eight areas of spots. Behavioural data (activity) was used to perform a classification process (TwoStep Cluster) dividing data into clusters. Following classification, Mann-Whitney $U$-test or Kruskal-Wallis comparisons were used to analyse all data, including the Fulton condition factor calculated by the formula $\mathrm{K}=100 * \mathrm{M}_{\mathrm{B}} \mathrm{L}_{\mathrm{F}}^{-3}$ and the different size classes. All statistics were performed in IBM SPSS Statistics 19 (IBM Corporation, www.ibm.com) and data are presented as mean \pm S.E if not stated otherwise.

\section{RESULTS}

\section{CAROTENOID PIGMENTATION}

Carotenoid-based pigmentation, expressed as spots $\mathrm{cm}^{-2}$, among the eight different areas were strongly positively correlated (Spearman correlation, all $P$-values $<0.01$; Table I). There was no difference between the size categories in the aggragate carotenoid-based pigmentation. 
Table I. The Spearman correlation coefficients for number of spots $\mathrm{cm}-2$ in each area and an aggregate for all the areas (areas 1-8), and the mean \pm s.e. for each area and the aggregate

\begin{tabular}{|c|c|c|c|c|c|c|c|c|c|c|}
\hline Area & 1 & 2 & 3 & 4 & 5 & 6 & 7 & 8 & $1-8$ & Spots $\mathrm{cm}^{-2}$ \\
\hline 1 & 1.00 & & & & & & & & & $0.74 \pm 0.15$ \\
\hline 2 & $0.69 * *$ & 1.00 & & & & & & & & $2.47 \pm 0.27$ \\
\hline 3 & $0.58 * *$ & $0.73 * *$ & 1.00 & & & & & & & $3.64 \pm 0.30$ \\
\hline 4 & $0.52 * *$ & $0.67 * *$ & $0.79 * *$ & 1.00 & & & & & & $4.21 \pm 0.41$ \\
\hline 5 & $0.45 * *$ & $0.55^{* *}$ & $0.49 * *$ & $0.62 * *$ & 1.00 & & & & & $2.18 \pm 0.14$ \\
\hline 6 & $0.42 * *$ & $0.57 * *$ & $0.70 * *$ & $0.65 * *$ & $0.71 * *$ & 1.00 & & & & $2.70 \pm 0.15$ \\
\hline 7 & $0.45 * *$ & $0.62 * *$ & $0.72 * *$ & $0.74 * *$ & $0.67 * *$ & $0.80 * *$ & 1.00 & & & $1.79 \pm 0.13$ \\
\hline 8 & $0.38 * *$ & $0.41 * *$ & $0.37 * *$ & $0.66 * *$ & $0.53 * *$ & $0.44 * *$ & $0.57 * *$ & 1.00 & & $2.36 \pm 0.26$ \\
\hline $1-8$ & $0.64 * *$ & $0.81 * *$ & $0.84 * *$ & $0.93 * *$ & $0.74 * *$ & $0.78 * *$ & $0.84 * *$ & $0.69 * *$ & 1.00 & $2.51 \pm 0.18$ \\
\hline
\end{tabular}

**Significant at 0.01 level (Spearman correlation).

\section{CLASSIFICATION OF DIFFERENT BEHAVIOURAL TYPES}

Activity, based on number of movements between the dark and light compartments was used to classify behavioural types. This resulted in one high-activity group $(6.46 \pm 0.83 \mathrm{nr}, \mathrm{n}=13)$ and one low-activity group $(1.09 \pm 0.11 \mathrm{nr}, \mathrm{n}=47$; Mann-Whitney U-test, $P<0.001)$.

\section{BEHAVIOUR, PHYSIOLOGY AND CAROTENOID-BASED PIGMENTATION IN HIGH ACTIVITY AND LOW ACTIVITY INDIVIDUALS}

The two different classes based on activity were significantly different in behaviour, physiology, and pigmentation (Fig. 2). High-activity individuals had a lower latency to enter an open field area (Mann-Whitney U-test $=439.0, P<0.05)$ and higher plasma cortisol levels (Mann-Whitney U-test $=185.0, P<0.05$ ) than low-activity individuals. Further, high-activity individuals also had a significantly higher number of spots $\mathrm{cm}^{-2}$ (Mann-Whitney U-test $=189.0, P<0.05)$ than low-activity individuals, and this difference was emphasized in area 2 (Mann-Whitney U-test $=131.0, P<0.01$ ).

The value of $\mathrm{K}$ ranged from 1.43 to 2.18 , with no difference between high-activity and low-activity individuals within the size categories. The different size classes, however, differed in $\mathrm{K}$ (Kruskal-Wallis $=10.682$, d.f $=2, P<0.01$ ). The large individuals had higher $\mathrm{K}$ than both small (Kruskal-Wallis pair-wise comparison $=-15.721, P<0.05$ ) and medium individuals (Kruskal-Wallis pair-wise comparison $=-14.493, P<0.05$ ).

\section{DISCUSSION}

\section{BEHAVIOURAL CLASSIFICATION}

In this study, S. alpinus were classified based on exploratory behaviour, thus creating groups of high- and low- activity individuals. These high- and low-activity individuals differed in behaviour, plasma cortisol and carotenoid skin pigmentation (i.e. number of spots).

Behavioural differences between high- and low-activity individuals consisted of both 
exploration and latency to enter a novel area. This suggests that the behaviours are linked. Five different axes including shyness-boldness, exploration-avoidance, activity, sociability and aggressiveness, were suggested by Réale et al. (2007) that could be used for temperament, personality and behavioural syndrome studies. Using this terminology, latency to enter a novel area could be exploring. Swimming between compartments could also be exploring. A high general activity, however, could also lead to a shorter latency to enter a novel area as well as more swimming between compartments. In either case, behaviour could be used to determine at least one of these behavioural aspects of temperament, personality, and behavioural syndrome in individual fishes.

Exploration

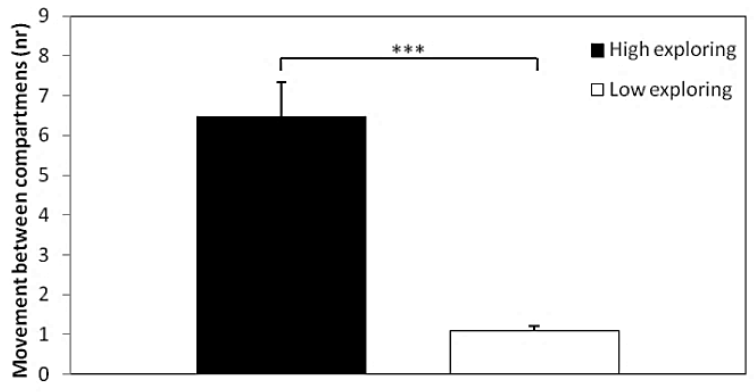

Latency

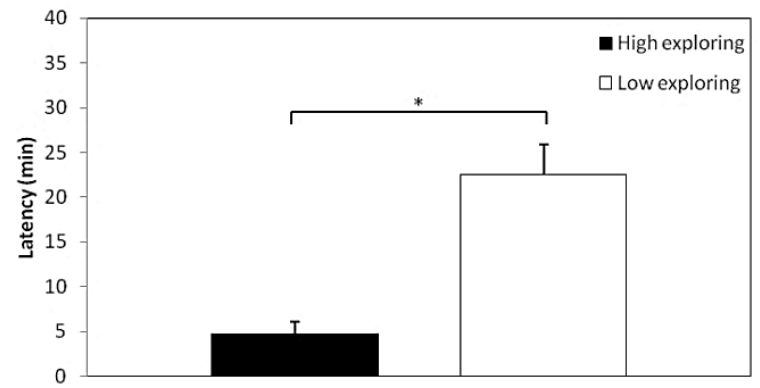

Plasma cortisol

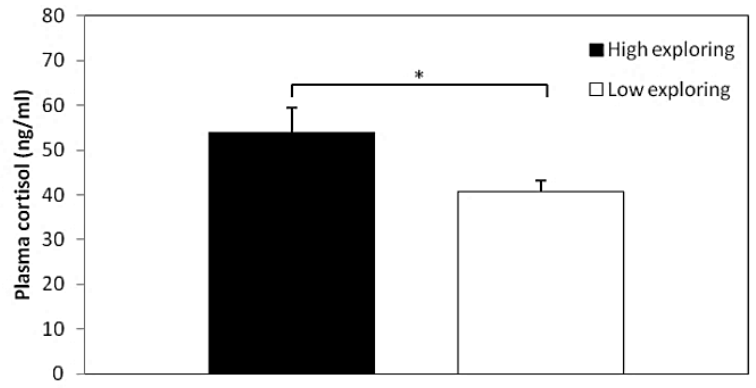

Pigmentation

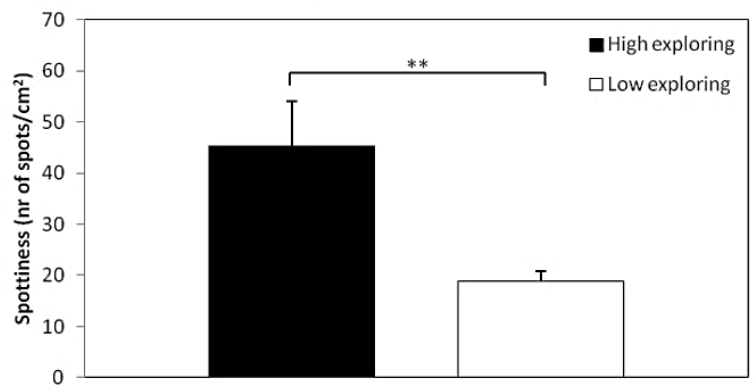

Fig. 2. The effect of classification into high-activity $(n=13)$ and low-activity $(n=47)$ individuals upon (a) exploration (movements), (b) latency to enter novel area, (c) plasma cortisol and (d) pigmenta- tion. Values are mean \pm s.e. and asterisks indicate difference between high exploring and low exploring individuals $(* P<0.05$; $* * P<$ $0.01 ; * * * P<0.001$; Mann - Whitney $U$-test).

\section{STRESS COPING STYLES}

Behaviour coupled to stress responsiveness in animals has been termed stress-coping styles (Koolhaas et al., 1999). In this study, a connection between behaviour and stress responsiveness was shown, with high-activity individuals having higher plasma cortisol levels following stress than low-activity individuals. Generally, high stress responsiveness is indicating a reactive stress coping style, i.e. individuals being less aggressive and less active (Koolhaas et al., 1999). Previous studies in both S. salar and HR and LR O. mykiss have shown that high stress responsiveness leads to lower activity during acute stress (Kittilsen $e t$ al., 2009; Backström et al., 2011). In this study behaviour was not monitored during acute stress because $S$. alpinus had been acclimatized for 1 day before the exploration test. In an earlier study, LR $O$. mykiss were more active immediately after exposure to an open field test (during the first 3-5 min) and then settled down, whereas HR O. mykiss appeared to remain active (Schjolden et al., 2005b). In this study, as the exploratory experiment was not an acute 
stressor, it appears likely that high-activity individuals were individuals remaining active over a longer time frame. Thus, it seems as high- and low-activity individuals appear to fit well with reactive and proactive coping style, respectively.

\section{CAROTENOID-BASED PIGMENTATION}

In this study, the carotenoid pigmentation appeared to be regularly distributed within each individual and a specific area could be used to predict the general pigmentation. Carotenoids are not synthesized in animals so they have to be obtained from diet. Ingested carotenoids have several functions including antioxidant activity, being the vitamin A precursor, and ornamental coloration (Svensson \& Wong, 2011). The ornamental colouration of carotenoids as a signalling mechanism has been studied extensively (Svensson \& Wong, 2011), and most of it has been concerned with sexual signalling. There have also been studies establishing a link between carotenoid pigmentation and physical prowess. For instance, in male $P$. reticulata, carotenoid pigmentation is positively correlated to swimming performance (Nicoletto, 1991). Male firemouth cichlids Cichlasoma meeki Brind 1918 fed a high carotenoid diet won more contest compared to males fed a low carotenoid diet (Evans \& Norris, 1996). Similarly, in a dyadic contest between male three-spined stickleback Gasterosteus aculeatus L. 1758, losers with higher carotenoid levels than winners took a longer time to lose contests (Guderley \& Couture, 2005). In zebra finches Taeniopygia guttata, carotenoid-supplemented diet enhanced flight performance (Blount \& Matheson, 2006). All of these studies indicate that carotenoid coloration is coupled to better physical performance. Thus, the result that high activity individuals have more carotenoid spots than low activity individuals indicates that they have a better physical status. Additionally, it is worth noting that in this study S. alpinus were all fed the same diet, and thus there should be no difference in dietary provision of carotenoids.

\section{CAROTENOIDS, STRESS COPING STYLES AND IMMUNE SYSTEM}

In $S$. salar and $O$. mykiss, melanin skin pigmentation has been connected to stress responsiveness (Kittilsen et al., 2009). In this study, a similar connection was noted between carotenoid skin pigmentation and stress responsiveness as well as behaviour, or stress coping style. Thus, it appears that carotenoid pigmentation in S. alpinus could be linked to stresscoping style. Furthermore, proactive juvenile $S$. salar have a higher number of melanin skin pigmentation and also harboured fewer female sea lice Lepeophtheirus salmonis carrying egg sacs than reactive $S$. salar with a low number of melanin skin pigmentation (Kittilsen et al., 2012). Thus, a connection between immune system and stress-coping style appears to be evident by pigmentation analysis. As carotenoid involvement in immune system is similar to melanin in some ways (Griffith et al., 2006), the observed differences in this study could possibly be linked to the immune system as well. This remains to be investigated.

Carotenoid pigmentation in S. alpinus could indicate more dietary carotenoid intake. All S. alpinus, however, were fed the same feed and K did not differ between high- and lowactivity individuals. Therefore, the differences in pigmentation could either be differences in health status or some underlying difference in carotenoid metabolism. Specific tests for health status were not performed, but all individuals appeared healthy by visual inspection and their values of K supported this. Thus, following the same reasoning as for $S$. salar, where high pigmentation and low stress reaction indicated a better immune response, it is likely that individuals in this study with fewer pigment spots would have a better immune 
response. This implies a difference in usage of dietary intake of carotenoids, with some $S$. alpinus using more for the immune system whereas others use more for skin pigmentation.

In conclusion, this study indicates that behaviour and stress responsiveness are correlated in $S$. alpinus, indicating different stress coping styles. The different stress coping styles also appear to be linked to carotenoid-based pigmentation in S. alpinus. These interesting results warrant further investigation to clarify the underlying mechanisms driving this connection between behaviour and physiology.

The methodology of this study was approved by the Swedish University of Agricultural Sciences Ethical Committee. Financing was granted by the Swedish Research Council Formas. We thank L Brännäs, H Carlberg, and B-S Wiklund for participating in the sampling process.

\section{References}

Backström, T., Schjolden, J., Øverli, Ø., Thörnqvist, P. O. \& Winberg, S. (2011). Stress effects on AVT and CRF systems in two strains of rainbow trout (Oncorhynchus mykiss) divergent in stress responsiveness. Hormones and Behavior 59, 180-186.

Barreto, R. E. \& Volpato, G. L. (2011). Ventilation rates indicate stress-coping styles in Nile tilapia. Journal of Biosciences (Bangalore) 36, 851-855.

Blount, J. D. \& Matheson, S. M. (2006). Effects of carotenoid supply on escape flight responses in zebra finches, Taeniopygia guttata. Animal Behaviour 72, 595-601.

Boissy, A. (1995). Fear and fearfulness in animals. Quarterly Review of Biology 70, 165-191.

Brelin, D., Petersson, E. \& Winberg, S. (2005). Divergent stress coping styles in juvenile brown trout (Salmo trutta). In Trends in Comparative Endocrinology and Neurobiology, pp. 239-245.

Burns, J. G. (2008). The validity of three tests of temperament in guppies (Poecilia reticulata). Journal of Comparative Psychology 122, 344-356.

Ducrest, A.-L., Keller, L. \& Roulin, A. (2008). Pleiotropy in the melanocortin system, coloration and behavioural syndromes. Trends in Ecology \& Evolution 23, 502-510.

Evans, M. R. \& Norris, K. (1996). The importance of carotenoids in signaling during aggressive interactions between male firemouth cichlids (Cichlasoma meeki). Behavioral Ecology 7, 1-6.

Gosling, S. D. (2001). From mice to men: What can we learn about personality from animal research? Psychological Bulletin 127, 45-86.

Griffith, S. C., Parker, T. H. \& Olson, V. A. (2006). Melanin- versus carotenoid-based sexual signals: is the difference really so black and red? Animal Behaviour 71, 749-763.

Guderley, H. \& Couture, P. (2005). Stickleback Fights: Why Do Winners Win? Influence of Metabolic and Morphometric Parameters. Physiological and Biochemical Zoology 78, 173-181.

Höglund, E., Balm, P. \& Winberg, S. (2000). Skin darkening, a potential social signal in subordinate Arctic charr (Salvelinus alpinus): the regulatory role of brain monoamines and pro-opiomelanocortin-derived peptides. Journal of Experimental Biology 203, 1711-1721.

Höglund, E., Balm, P. H. M. \& Winberg, S. (2002). Behavioural and neuroendocrine effects of environmental background colour and social interaction in Arctic charr (Salvelinus alpinus). Journal of Experimental Biology 205, 2535-2543. 
Kittilsen, S., Johansen, I. B., Braastad, B. O. \& Øverli, Ø. (2012). Pigments, parasites and personalitiy: towards a unifying role for steroid hormones? PLoS One 7, e34281.

Kittilsen, S., Schjolden, J., Beitnes-Johansen, I., Shaw, J. C., Pottinger, T. G., Sorensen, C., Braastad, B. O., Bakken, M. \& Øverli, Ø. (2009). Melanin-based skin spots reflect stress responsiveness in salmonid fish. Horm Behav 56, 292-298.

Klemetsen, A., Amundsen, P. A., Dempson, J. B., Jonsson, B., Jonsson, N., O'Connell, M. F. \& Mortensen, E. (2003). Atlantic salmon Salmo salar L., brown trout Salmo trutta L. and Arctic charr Salvelinus alpinus (L.): a review of aspects of their life histories. Ecology of Freshwater Fish 12, 1-59.

Kodric-Brown, A. \& Nicoletto, P. (2005). Courtship behavior, swimming performance, and microhabitat use of Trinidadian guppies. Environmental Biology of Fishes 73, 299307.

Koolhaas, J. M., de Boer, S. F., Buwalda, B. \& van Reenen, K. (2007). Individual Variation in Coping with Stress: A Multidimensional Approach of Ultimate and Proximate Mechanisms. Brain, Behavior and Evolution 70, 218-226.

Koolhaas, J. M., Korte, S. M., De Boer, S. F., Van Der Vegt, B. J., Van Reenen, C. G., Hopster, H., De Jong, I. C., Ruis, M. A. W. \& Blokhuis, H. J. (1999). Coping styles in animals: current status in behavior and stress-physiology. Neuroscience \& Biobehavioral Reviews 23, 925-935.

Kristiansen, T. S. \& Fernö, A. (2007). Individual behaviour and growth of halibut (Hippoglossus hippoglossus L.) fed sinking and floating feed: evidence of different coping styles. Applied Animal Behaviour Science 104, 236-250.

Nicoletto, P. F. (1991). The relationship between male ornamentation and swimming performance in the guppy, Poecilia reticulata. Behavioral Ecology and Sociobiology 28, 365-370.

Nicoletto, P. F. \& Kodric-Brown, A. (1999). The Relationship Among Swimming Performance, Courtship Behavior, and Carotenoid Pigmentation of Guppies in Four Rivers of Trinidad. Environmental Biology of Fishes 55, 227-235.

Nilsson, J., Brännäs, E. \& Eriksson, L. O. (2010). The Swedish Arctic charr breeding programme. Hydrobiologia 650, 275-282.

Øverli, Ø., Sørensen, C., Pulman, K. G. T., Pottinger, T. G., Korzan, W., Summers, C. H. \& Nilsson, G. E. (2007). Evolutionary background for stress-coping styles: Relationships between physiological, behavioral, and cognitive traits in nonmammalian vertebrates. Neuroscience \& Biobehavioral Reviews 31, 396-412.

Pottinger, T. G. \& Carrick, T. R. (1999). Modification of the plasma cortisol response to stress in rainbow trout by selective breeding. General and Comparative Endocrinology 116, 122-132.

Pottinger, T. G. \& Carrick, T. R. (2001). Stress responsiveness affects dominant-subordinate relationships in rainbow trout. Hormones and Behavior 40, 419-427.

Reale, D., Reader, S. M., Sol, D., McDougall, P. T. \& Dingemanse, N. J. (2007). Integrating animal temperament within ecology and evolution. Biological Reviews (Cambridge) 82, 291-318.

Schjolden, J., Stoskhus, A. \& Winberg, S. (2005a). Does individual variation in stress responses and agonistic behavior reflect divergent stress coping strategies in juvenile rainbow trout? Physiological and Biochemical Zoology 78, 715-723.

Schjolden, J., Backström, T., Pulman, K. G. T., Pottinger, T. G. \& Winberg, S. (2005b). Divergence in behavioural responses to stress in two strains of rainbow trout (Oncorhynchus mykiss) with contrasting stress responsiveness. Hormones and Behavior 48, 537-544. 
Sih, A., Bell, A. \& Johnson, J. C. (2004). Behavioral syndromes: an ecological and evolutionary overview. Trends in Ecology \& Evolution 19, 372-378.

Silva, P. I. M., Martins, C. I. M., Engrola, S., Marino, G., Øverli, Ø. \& Conceição, L. E. C. (2010). Individual differences in cortisol levels and behaviour of Senegalese sole (Solea senegalensis) juveniles: Evidence for coping styles. Applied Animal Behaviour Science 124, 75-81.

Svensson, P. A. \& Wong, B. B. M. (2011). Carotenoid-based signals in behavioural ecology: a review. Behaviour 148, 131-189. 